\title{
SEARCH FOR HIGGS AND NEW PHENOMENA AT COLLIDERS
}

\author{
STEPHAN LAMMEL \\ Fermi National Accelerator Laboratory, Batavia, Illinois 60510, USA \\ E-mail: lammel@fnal.gov
}

\begin{abstract}
The present status of searches for the Higgs boson(s) and new phenomena is reviewed. The focus is on analyses and results from the current runs of the HERA and Tevatron experiments. The LEP experiments have released their final combined MSSM Higgs results for this conference. Also included are results from sensitivity studies of the LHC experiments and lepton flavour violating searches from the $\mathrm{B}$ factories, KEKB and PEP-II.
\end{abstract}

\section{Introduction}

A scalar Higgs particle ${ }^{1}$ has been postulated over 30 years ago as the mechanism of electroweak symmetry breaking in the Standard Model (SM) of particle physics. This spontaneous breaking introduces a huge hierarchy between the electroweak and Planck scales that is unsatisfying. Extensions to the SM have been proposed over the years to avoid unnatural finetuning. Supersymmetry ${ }^{2}$ (SUSY) is one such attractive extensions. Depending on its internal structure and SUSY breaking mechanism, a variety of new phenomena are expected to be observed. Rare signatures, as in high-mass tails or from SM suppressed processes, are good places for generic beyond-the-Standard Model searches.

The Large Electron Positron (LEP) collider at CERN completed operation about four years ago. It ran at a center-ofmass energy of up to $209 \mathrm{GeV}$ and delivered about $1 \mathrm{fb}^{-1}$ of data to the four experiments, ALEPH, DELPHI, L3, and OPAL. The data are analysed. Extensive searches for Higgs and new phenomena have come up negative. For many new particles coupling to the $\mathrm{Z}$ boson LEP still holds the most stringent limits.

Two machines, the Hadron Electron Ring Accelerator (HERA) and the Tevatron, are currently running at the energie frontier with ever increasing luminosities. HERA at DESY collides electrons or positrons with protons at a center-of-mass energy of $319 \mathrm{GeV}$. The HERA upgrade increased the luminosity by a factor of 4.7. So far the machine has delivered over $180 \mathrm{pb}^{-1}$ of electron-proton (about half) and positron-proton data to the two experiments, H1 and ZEUS. The experiments are particularly sensitive to new particles coupling to electron/positron and up/down quarks. HERA II can also deliver polarized lepton beams.

The Fermilab Tevatron collides proton and antiprotons at a center-of-mass energy of $1.96 \mathrm{TeV}$. Luminosity upgrades are continuing. So far the machine has delivered over $1 \mathrm{fb}^{-1}$ of data to the two experiments, CDF and $\mathrm{D} \varnothing$. The improved detectors, higher cener-of-mass energy, and ten fold increase in luminosity enable the experiments not only to significantly extend previous searches but provide them with a substantial discovery potential.

The Large Hadron Collider (LHC) at CERN and the International Linear Collider (ILC) are machines under construction and in the planning phase. The LHC will collide protons with protons at a center-of-mass energy of $14 \mathrm{TeV}$. First collisions are expected in 2007. The two experiments, ATLAS and CMS, have made detailed studies of their reach to new physics. LHC is expected to boost our sensitivity to new physics by an order of magnitude in energy/mass. The ILC will collide electrons and positrons with a center-of-mass energy of several hundred 


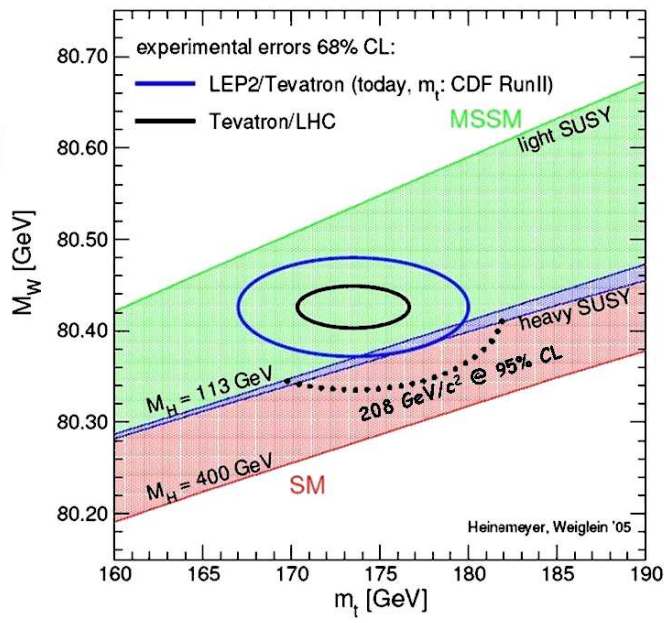

Figure 1. One sigma contours of the current $\mathrm{W}$ and top mass measurments compared to SM and MSSM Higgs masses. Plot from Heinemeyer ${ }^{4}$ updated for new CDF top mass measurment.

$\mathrm{GeV}$. It will be the next generation machine for precision measurments, like LEP was.

\subsection{Precision Electroweak and Top Measurments}

Precision electroweak measurments allow us to check the SM for consistency or derive the mass of the unknown Higgs particle. For Higgs prediction, the $\mathrm{W}$ boson mass and top quark mass are key ingredients. With the new preliminary CDF Run II top mass measurment ${ }^{3}$, the world average is pulled down to $\mathrm{m}_{\mathrm{t}}=174.3 \pm 3.4 \mathrm{GeV} / c^{2}$. Figure 1 shows the $1 \sigma$ and $95 \%$ confidence level (CL) contours of the $\mathrm{W}$ and top mass with overlaid Higgs mass. Current measurments put the SM Higgs below $208 \mathrm{GeV} / c^{2}$ at $95 \% \mathrm{CL}$.

However, the top mass is an even more important ingredient for the Higgs in Minimal Supersymmetric extensions of the Standard Model (MSSM). The MSSM exclusion at low $\tan (\beta)$ derived from the SM Higgs limit of LEP depends very sensitively on the mass of the top quark and vanishes when the top mass is large.

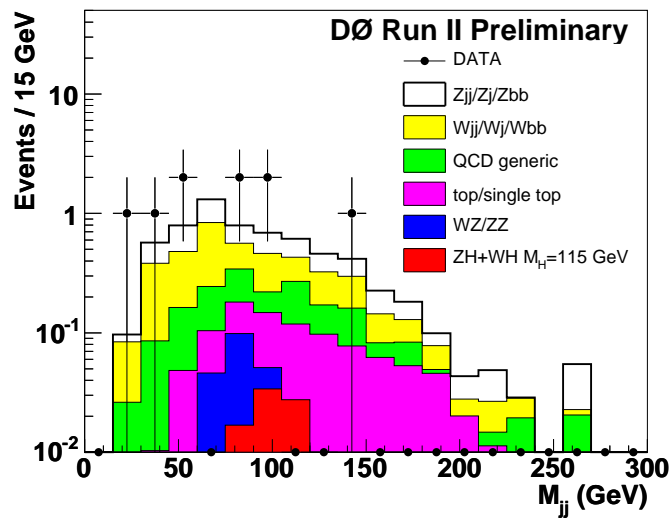

Figure 2. Dijet mass spectrum of the $\mathrm{D} \varnothing \mathrm{ZH}$ analysis after two b-tags.

\section{Standard Model Higgs}

The current lower limit on the Higgs mass of $114.4 \mathrm{GeV} / c^{2}$ at $95 \% \mathrm{CL}$ comes from the LEP experiments $^{5}$. They did a fantastic job of pushing the Higgs mass limit well above the $\mathrm{Z}$ pole where it would be hard for proton-antiproton experiments to detect. The Tevatron is the current place for Higgs searches with an expected sensitivity to about $130 \mathrm{GeV} / c^{2}$. Here the main Higgs production mechanism is via gluon-gluon fusion. Associated production with a $\mathrm{W}$ or $\mathrm{Z}$ has a factor five lower cross-section. For low Higgs masses, below $135 \mathrm{GeV} / c^{2}$, the $\mathrm{b} \overline{\mathrm{b}}$ decay mode is dominant. With a leptonic $\mathrm{W}$ or $\mathrm{Z}$ decay we get signatures of zero, one, or two charged leptons, an imbalance of energy in the transverse plane, missing $E_{\mathrm{T}}$ (in case of zero or one charged lepton), and two b-jets. For heavier Higgs the $\mathrm{WW}^{*}$ decay dominates and then Higgs production via gluon fusion yields a viable signature.

The WH analyses of CDF and DØ were performed early on and results are updated regularly with increased luminosity ${ }^{6}$. The DØ experiment has also completed a search in the $\mathrm{ZH}$ channel where the $\mathrm{Z}$ decays into neutrinos ${ }^{7}$. The analysis compares the missing $E_{\mathrm{T}}\left(B_{\mathrm{T}}\right)$ as calculated from all energy in 


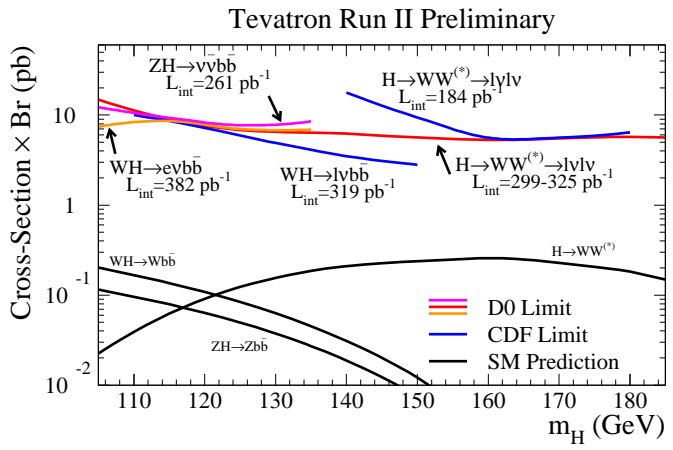

Figure 3. Current CDF and DØ Run II Higgs crosssection times branching ratio limits from the $\mathrm{WH}$, $\mathrm{ZH}$, and $\mathrm{WW}^{*}$ channel.

the detector with the calculation from just clustered energy and the jet energy vector sum with the track momentum vector sum to reduce instrumental background which comes mainly from jet mismeasurments. The main background in the analysis comes from $\mathrm{Z}$ plus multijet production and $\mathrm{W}$ plus $\mathrm{b} \overline{\mathrm{b}}$ production with $\mathrm{W}$ decay into $\tau \nu$ decay. Figure 2 shows the dijet mass spectrum after two btags are required. No excess of events over background expectation is observed in this search nor in any other Higgs analysis of CDF and $\mathrm{D} \varnothing$. The cross-section times branching ratio limit of this analysis is shown in Fig. 3 together with the limits from the other Tevatron SM Higgs searches.

The sensitivity of CDF and DØ is currently between 3 and $10 \mathrm{pb}$ while a SM Higgs is at about $0.2 \mathrm{pb}$. The difference between the current and the final Run II Higgs sensitivity projection ${ }^{8}$ is understood. In addition to the luminosity accumulation, improvements in lepton and b-tagging acceptance, the dijet mass resolution, and analysis techniques will bring the sensitivity of the experiments to the projections made before Run II.

At LHC the Higgs production crosssection is huge. Even a decay mode with small branching ratio, like Higgs into a photon pair, yields a sizable event number. The two experiments each have an electromag-

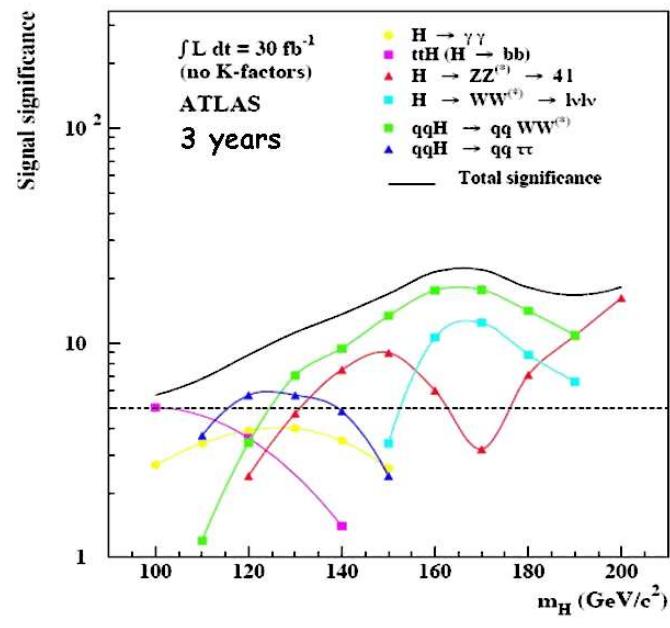

Figure 4. SM Higgs signal significance of the ATLAS experiment for the different search channels.

netic calorimeter with very precise energy resolution to be able to observe a diphoton mass bump from Higgs ${ }^{9}$ on top of the huge diphoton continuum. For LHC vector boson fusion, however, will be the most important production for Higgs. Both ATLAS and CMS can observe a SM Higgs up to several hundred $\mathrm{GeV} / c^{2}$ after a few years of running, Fig. 4. For LHC the observation of a Higgs boson would be just the initial step. The two experiments can measure the ratio of couplings and decay widths to an uncertainty of 20 and $30 \%$.

\section{MSSM Higgs}

Current and next generation experiments cover a SM Higgs well. The Higgs sector, however, can be richer than a single doublet. Supersymmetry extends the symmetry concept, that has been so successfull in particle physics, to the spin sector. It provides a consistent framework for gauge unification and solves the hierarchy problem of the SM. No SUSY particles have been observed so far. Several SUSY breaking scenarios are under consideration which determine the SUSY structure. The MSSM is the general mini- 


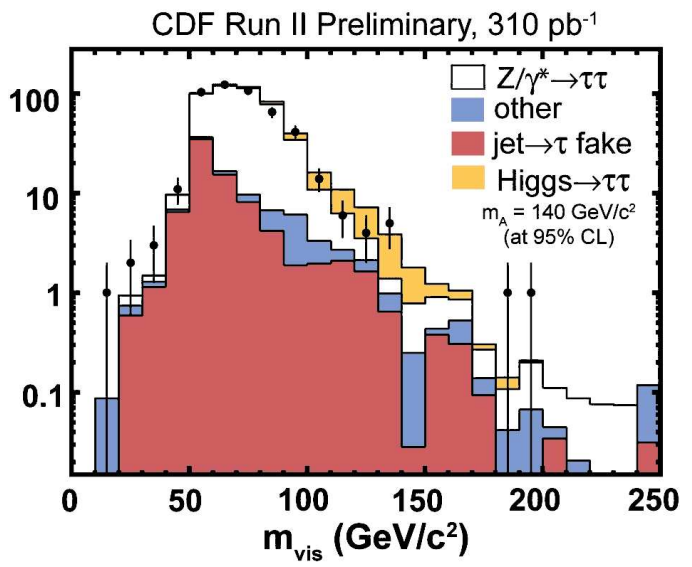

Figure 5. Distribution of the "visible" Higgs mass in the CDF ditau analysis.

mal supersymmetric extension of the SM. It has two Higgs doublets yielding five physical Higgs particles: h, $\mathrm{H}, \mathrm{A}, \mathrm{H}^{+}$, and $\mathrm{H}^{-}$. At tree level the Higgs sector is described by two parameters, the pseudoscalar Higgs mass, $\mathrm{m}_{\mathrm{A}}$, and ratio of the two Higgs vacuum expectation values, $\tan (\beta)$. The MSSM, although the minimal extension, has a lot of free parameters. One normally uses models constrained based on SUSY breaking scenarios and GUT scale relations or special benchmarking models.

At the Tevatron the Higgses of the MSSM are of particular interest. The Yukawa coupling to down-type fermions is enhanced, boosting the cross-section by a factor of $\tan (\beta)^{2}$. For large $\tan (\beta)$ the pseudoscalar Higgs and either $\mathrm{h}$ or $\mathrm{H}$ are expected to be almost mass degenerate. The branching ratio into $\mathrm{b} \overline{\mathrm{b}}$ is at around $90 \%$ independent of mass. Decays into tau pairs account for close to $10 \%$.

Two neutral MSSM Higgs searches are performed at CDF and D $\varnothing$. The first is based on Higgs plus $\mathrm{b} \overline{\mathrm{b}}$ production: $\mathrm{b} \overline{\mathrm{b}} \mathrm{A} \rightarrow \mathrm{b} \overline{\mathrm{b}} \mathrm{b} \overline{\mathrm{b}}$. It yields a striking four b-jet signature. The second search is based on the tau decay mode: $\mathrm{A} \rightarrow \tau^{+} \tau^{-}$.

Tau leptons are not as easily identified

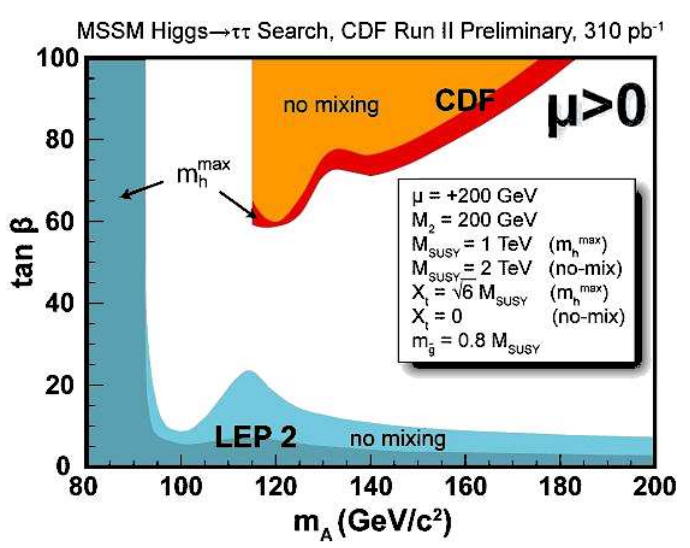

Figure 6. Excluded regions in the mass of A versus $\tan (\beta)$ plane for the $\mathrm{m}_{\mathrm{h}}^{\max }$ and no mixing scenario for the Higgsino mass marameter $\mu>0$.

as electrons or muons. The CDF analysis ${ }^{10}$ is based on one leptonic tau decay and one hadronic tau decay. Jets from hadronic tau decays are very narrow, pencil like, compared to quark/gluon jets. CDF uses a double cone algorithm to identify hadronic tau decays. An efficiency of $46 \%$ is achieved with a misidentification rate between $1.5 \%$ to $0.1 \%$ per jet depending on the jet energy. For the Higgs search the experiment uses a data sample selected by an electron or muon plus track trigger to achieve high efficiency. Figure 5 shows the visible mass of the ditau system, calculated from the momentum vector of the lepton, hadronic tau, and $\xi_{\mathrm{T}}$. The main background comes from Z and DrellYan ditau production. No excess of events is observed in the first $310 \mathrm{pb}^{-1}$ of Run II data. A binned likelihood fit in the visible mass is used to set limits on the mass of A versus $\tan (\beta)$, Fig 6 .

The $D \varnothing$ analysis ${ }^{11}$ for the four b-jet channel requires three b-tag jets in the event. The first jet has to have $E_{\mathrm{T}}>35 \mathrm{GeV}$ while the third can be as low as $15 \mathrm{GeV}$. To estimate the background from light quark and gluon jets, the probability of mis-tagging a jet is measured on the three jet sample before b-tagging, subtracting any true heavy 


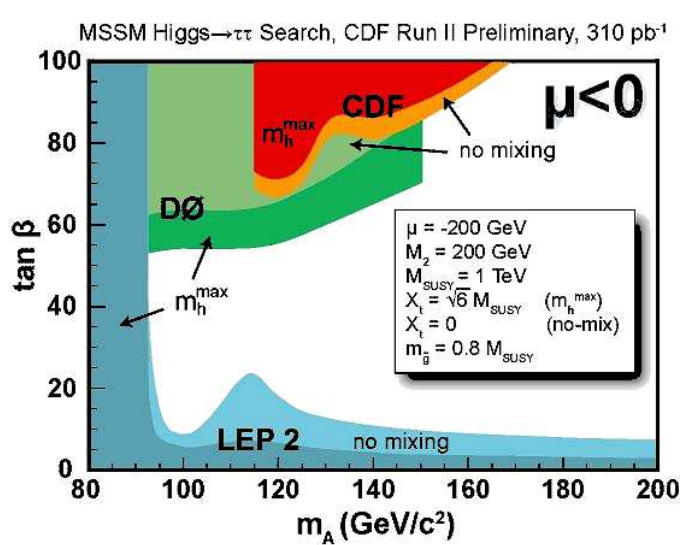

Figure 7. Excluded regions in the mass of A versus $\tan (\beta)$ plane for the $\mathrm{m}_{\mathrm{h}}^{\max }$ and no mixing scenario for the Higgsino mass marameter $\mu<0$.

flavour contribution. Those mistag functions are then applied to the untagged jets in the double b-tag sample to get the shape of the multijet background to the triple b-tag sample. D $\varnothing$ determines the overall background normalization by fitting the dijet mass outside the hypothesized signal region in the triple b-tag sample. Figure 7 shows the mass versus $\tan (\beta)$ limit obtained by this analysis. For $\mu>0$ the sensitivity of the four b-jet channel is very low due to the lower cross-section and lower branching ratio into $\mathrm{b} \overline{\mathrm{b}}$, while for the tau channel cross-section reduction and branching ratio enhancement compensate.

The final combined MSSM Higgs mass limits from the four LEP experiments have been released ${ }^{12}$. There are no signals of Higgsstrahlung or pair production. Sensitivity is evaluated in several benchmark models. A top mass of $179 \mathrm{GeV} / c^{2}$ is assumed for all limits. Figure 8 shows the excluded mass of $\mathrm{A}$ versus $\tan (\beta)$ for the classic no-stop mixing benchmark model with $\mathrm{M}_{\mathrm{SUSY}}=1000 \mathrm{GeV} / c^{2}, \mathrm{M}_{2}=200 \mathrm{GeV} / c^{2}$, $\mu=-200 \mathrm{GeV} / c^{2}, \mathrm{~m}_{\text {gluino }}=800 \mathrm{GeV} / c^{2}$, $A=0+\mu \cdot \cot (\beta)$. The excluded area is reduced in the case of stop mixing and is quite sensitive to the top mass. The LEP

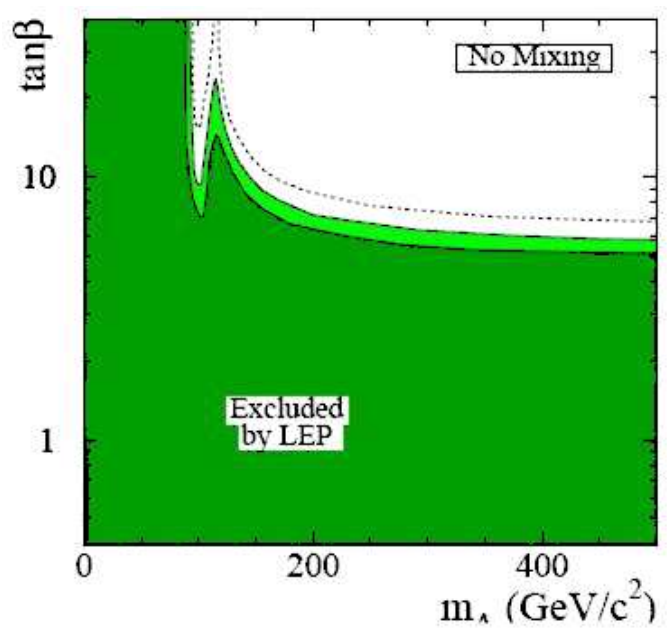

Figure 8. Excluded regions in the mass of A versus $\tan (\beta)$ plane for the no mixing scenario. Dark areas are excluded at over $99 \% \mathrm{CL}$, light areas at $95 \% \mathrm{CL}$.

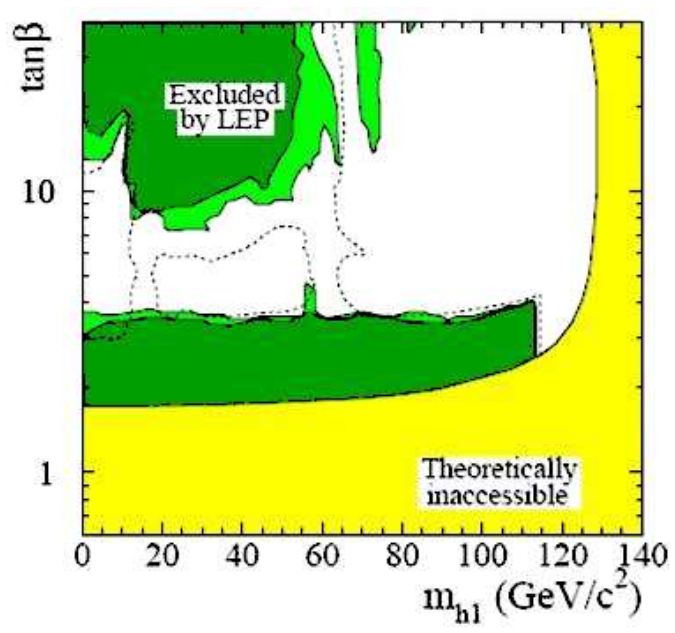

Figure 9. Excluded regions in the mass of $\mathrm{h}_{1}$ versus $\tan (\beta)$ plane for the CP-violating scenario. Dark areas are excluded at over $99 \%$ CL, light areas at $95 \%$ CL. 


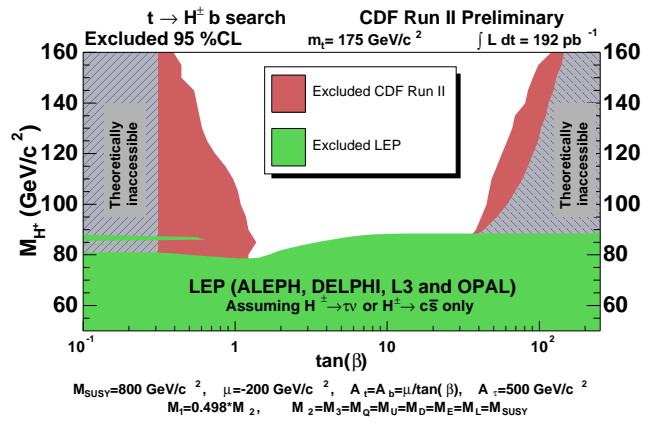

Figure 10. Excluded regions in the $\tan (\beta)$ versus mass of charged Higgs plane for a tevatron benchmarking scenario. The dark area is excluded at $95 \%$ $\mathrm{CL}$, the dark line shows the expected limit with $1 \sigma$ band.

experiments also considered the case of CPviolation in the Higgs sector. Such a scenario appeals in explaining the cosmic matterantimatter asymmetry. Experimentally such a scenario is much more challenging as the lightest Higgs can decouple from the Z. Figure 9 shows the LEP results. An inconsistency in the prediction from $\mathrm{CPH}$ and FeynHiggs for the $\mathrm{h}_{2} \rightarrow \mathrm{h}_{1} \mathrm{~h}_{1}$ branching ratio causes the hole at $\tan (\beta) \sim 6$ to open up $^{13}$.

There are also two charged Higgs particles in the MSSM. CDF uses its top crosssection measurments from the various decay channels to search for top decays into charged Higgs plus b-quark ${ }^{14}$. Such a decay would change the expected number of events differently in the dilepton, lepton plus single b-tag, lepton plus double b-tag, and lepton plus tau channel, especially for small and large $\tan (\beta)$ values. Figure 10 shows the excluded mass as function of $\tan (\beta)$ in one of the benchmark models studied.

\section{Supersymmetry}

From the LEP experiments ${ }^{15}$ we know that the chargino has to be heavier than $103.5 \mathrm{GeV} / c^{2}$. At the Tevatron the crosssection for chargino-neutralino production is rather small. However, in $R_{\mathrm{P}}$ con-
Table 1. Observed events and expected number of background events in the six channels of the $D \varnothing$ chargino-neutralino analysis.

\begin{tabular}{|l|c|c|}
\hline Channel & Expected & Observed \\
\hline e et & $0.21 \pm 0.12$ & 0 \\
\hline $\mathrm{e} \mu \mathrm{t}$ & $0.31 \pm 0.13$ & 0 \\
\hline$\mu \mu \mathrm{t}$ & $1.75 \pm 0.57$ & 2 \\
\hline$\mu^{ \pm} \mu^{ \pm}$ & $0.64 \pm 0.38$ & 1 \\
\hline $\mathrm{e} \tau_{\mathrm{h}} \mathrm{t}$ & $0.58 \pm 0.14$ & 0 \\
\hline$\mu \tau_{\mathrm{h}} \mathrm{t}$ & $0.36 \pm 0.13$ & 1 \\
\hline \hline Total & $3.85 \pm 0.75$ & 4 \\
\hline
\end{tabular}

serving minimal supergravity inspired SUSY (mSUGRA) one can get a very distinct signature. In case of leptonic chargino and neutralino decay, the event will contain only three charged leptons and missing $E_{\mathrm{T}}$ from the escaping neutrinos and the lightest SUSY particles (LSP). The challenge in the analysis is the charged lepton acceptance times efficiency since it enters with third power. For $\tan (\beta)$ values above 8 to 10 , tau decays become significant and tau identification thus very important.

The D $\varnothing$ analysis ${ }^{16}$ searches in six separate channels and combines the results. In all the channels known dilepton resonances are removed and a combined cut on the $E_{\mathrm{T}}$ and $p_{\mathrm{T}}$ of the third lepton used to suppress background from mainly misidentified leptons and diboson production. Table 1 shows the expected background and observed number of events in each of the six channels. In the $320 \mathrm{pb}^{-1}$ of data analysed, no excess is observed. DØ continues to set cross-section times branching ratio into three lepton limits. The analysis also improves the LEP chargino mass limit to $116 \mathrm{GeV} / c^{2}$ in case of light sleptons, i.e. small $\mathrm{m}_{0}$.

The production cross-section of coloured SUSY particles is much larger than that of chargino-neutralino. The squarks of the first two generations are assumed to be degenerate in mass. Stop and sbottom quarks could be significantly lighter due to the large top 


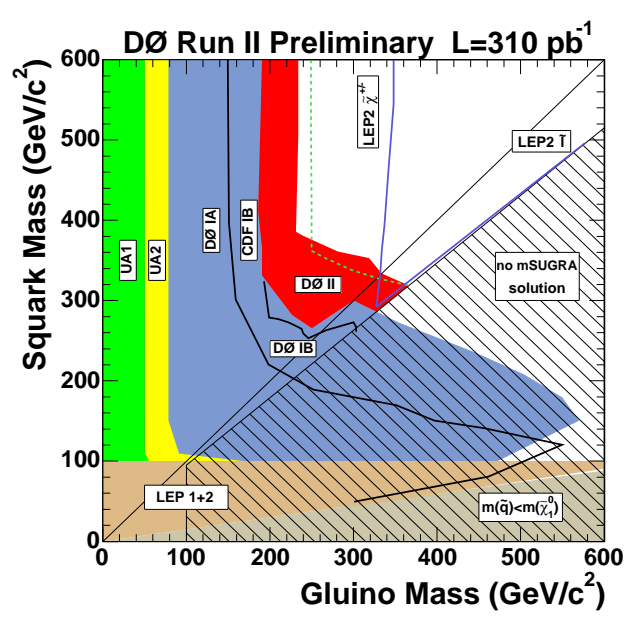

Figure 11. Excluded region in the gluino versus squark mass plane of the $\mathrm{D} \varnothing$ missing $E_{\mathrm{T}}$ plus multijet analysis.

Yukawa coupling. CDF and DØ have dedicated analyses for those ${ }^{17}$. The analyses assume direct decay of the third generation squark into LSP: $\tilde{b} \rightarrow \mathrm{b} \tilde{\chi}_{1}^{0}$ or $\tilde{\mathrm{t}} \rightarrow \mathrm{c} \tilde{\chi}_{1}^{0}$. Both direct production of $\tilde{\bar{t}} \overline{\tilde{t}}$ and $\tilde{\mathrm{b}} \overline{\mathrm{b}}$ and indirect production through, for instance, $\tilde{\mathrm{g}} \rightarrow \tilde{\mathrm{b}} \overline{\mathrm{b}}$ are researched.

For the gluinos and squarks of the first two generation the signature depends on the mass hierarchy. If the squarks are lighter, squark production is dominant and squarks will decay via $\tilde{\mathrm{q}} \rightarrow \mathrm{q} \tilde{\chi}^{0}$ or $\tilde{\mathrm{q}} \rightarrow \mathrm{q}^{\prime} \tilde{\chi}^{ \pm}$with $\tilde{\chi}^{ \pm} \rightarrow \mathrm{q} \overline{\mathrm{q}} \tilde{\chi}_{1}^{0}$. In case the gluino is the lighter one, gluino pair production is dominant with $\tilde{\mathrm{g}} \rightarrow \mathrm{q} \overline{\mathrm{q}} \tilde{\chi}^{0}$ or $\tilde{\mathrm{g}} \rightarrow \mathrm{q} \overline{\mathrm{q}}^{\prime} \tilde{\chi}^{ \pm}$. With the jets from the chargino decay generally being softer, this yields a 2,3 , or 4 jet signature together with missing $E_{\mathrm{T}}$. The $\mathrm{D} \varnothing$ analysis ${ }^{18}$ makes a preselection, vetoing jet back-to-back topologies, events with leptons, and events where the missing $E_{\mathrm{T}}$ is close in azimuthal angle to a jet. The main SM backgrounds are from $\mathrm{W} / \mathrm{Z}$ plus multijet and QCD multijet production. After the preselection dedicated analyses are made for each jet multiplicity. In all three cases the observed events are explained by the background estimate. Fig-

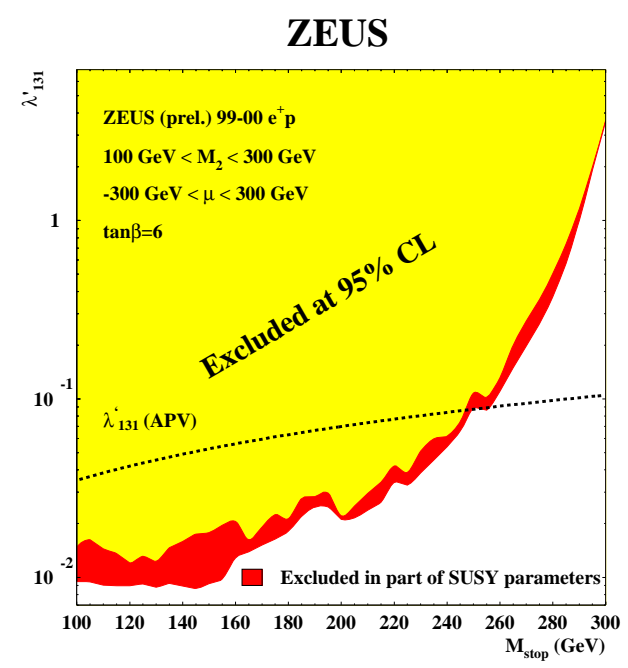

Figure 12. Excluded stop mass as function of $\lambda_{131}^{\prime}$ coupling of the ZEUS analysis.

ure 11 shows the new excluded region in gluino versus squark mass.

$R_{\mathrm{P}}$ conserving SUSY yields a natural dark matter candidate. However, $R$-parity conservation is really put into the models ad $h o c$ and nature may not conserve it. In case of $R_{\mathrm{P}}$ violation $\left(\not R_{\mathrm{P}}\right)$, different signatures arise. In the case of a non-vanishing $\lambda^{\prime}$ coupling electrons and $\mathrm{u} / \mathrm{d}$-quarks can couple, ideal for HERA. In electron-proton mode, $\lambda_{11 k}^{\prime}$ couplings are accessible while in positronproton mode, $\lambda_{1 j 1}^{\prime}$ couplings would produce resonant $\tilde{u}_{\mathrm{L}}$. Both $\mathrm{H} 1$ and ZEUS searched for a large variety of $\not h_{\mathrm{P}}$ signatures. The most striking signature is "wrong" sign electrons, i.e. events with energetic electrons while in positron-proton mode and events with positrons, jets, and no missing $E_{\mathrm{T}}$ while in electron-proton mode. No signals of $\not R_{\mathrm{P}}$ SUSY has been found. Figures 12 and 13 show the stop and sbottom mass limits as function of the coupling constant from ZEUS and $\mathrm{H} 1$. Squarks with $R_{\mathrm{P}}$ violating couplings of electroweak strength are excluded up to $275 \mathrm{GeV} / c^{2}$.

In case SUSY is broken via gauge interactions (GMSB) the gravition acquires 


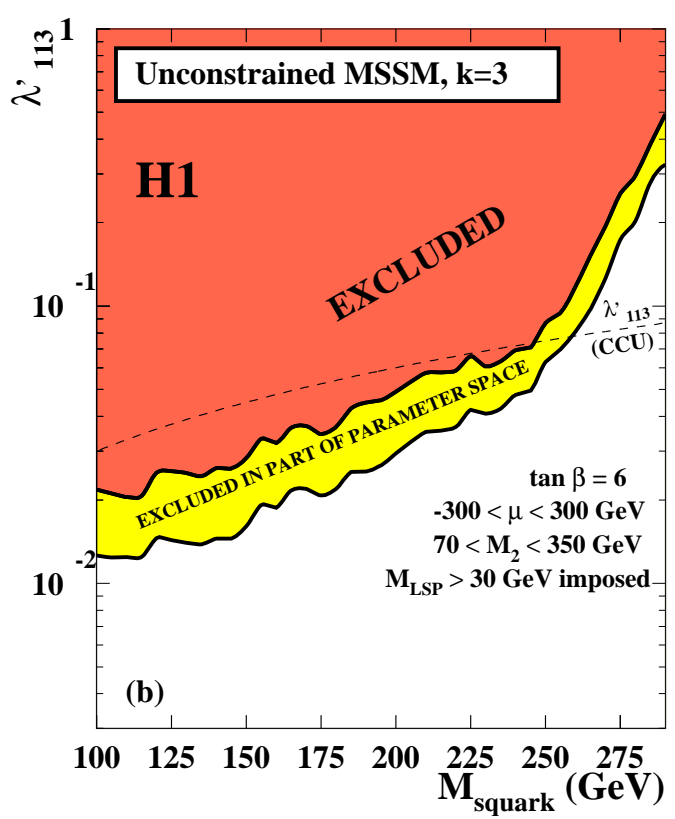

Figure 13. Excluded sbottom mass as function of $\lambda_{113}^{\prime}$ coupling of the $\mathrm{H} 1$ analysis.

a small mass and becomes the LSP. The next-to-lightest SUSY particle will decay into a photon plus gravitino for the distinct GMSB photon signature. CDF and D have both searched in the diphoton plus $Z_{\mathrm{T}}$ channel $^{20}$. The experiments use charginoneutralino production as reference model for the search. The two experiment have combined their results from the first $250 \mathrm{pb}^{-1}$ of data and exclude charginos in GMSB models below $209 \mathrm{GeV} / c^{2}$ at $95 \% \mathrm{CL}$.

In the case of low energy supersymmetry, LHC will be a great machine ${ }^{21}$. It will provide a definite answer to the question and with a small luminosity of only a few month probe SUSY scales of over a TeV. But LHC can do more and measure sparticle masses, for instance, for the second lightest neutralino from the dilepton spectrum endpoint or even the gluino mass from the topbottom endpoint.

The ILC ${ }^{22}$, however, will be required for precision mass and coupling measurements.

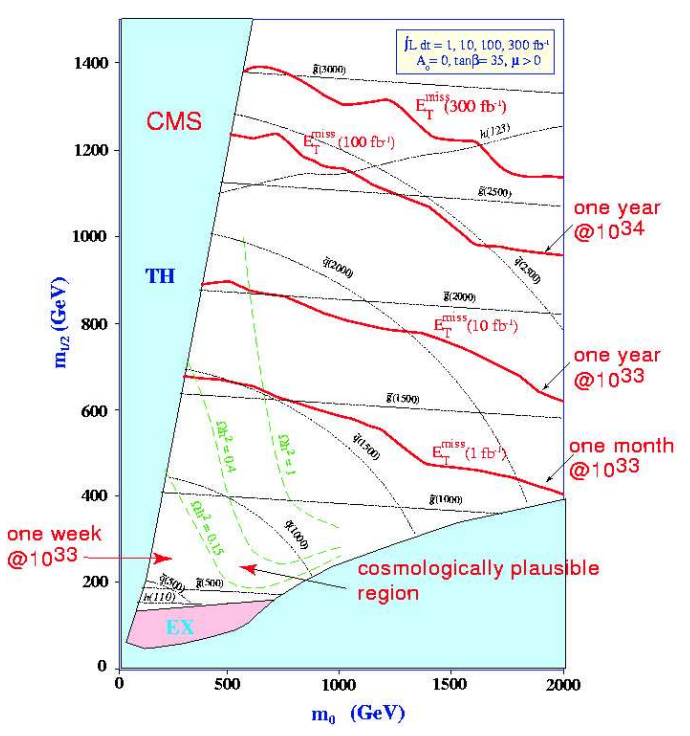

Figure 14. SUSY sensitivity of CMS in the $\mathrm{m}_{0}$ versus $\mathrm{m}_{1 / 2}$ plane for different integrated luminosity.

\section{Isolated Lepton and Missing Energy}

In Run I of HERA H1 observed an excess of events with isolated lepton $p_{\mathrm{T}}>10 \mathrm{GeV}$ and missing $E_{\mathrm{T}}>12 \mathrm{GeV}$ beyond what one would expect from $\mathrm{W}$ production ${ }^{23}$. The excess was pronounced at large $p_{\mathrm{T}}^{X}>25 \mathrm{GeV}$ and did not fit well any new physics model. Both H1 and ZEUS have searched for an isolated lepton plus $E_{\mathrm{T}}$ signature in the new Run II data ${ }^{24}$. H1 has used the identical selection in the analysis of the new data, separately for positron-proton and electronproton data. The muon channel shows no more the excess seen in Run I while an event excess remains in the electron channel. H1 has also analysed the tau data from Run I which show no excess either. ZEUS did not observe an event excess in Run I. With $40 \mathrm{pb}^{-1}$ of Run II data analysed ZEUS finds also no excess in the electron channel. Table 2 shows the current results of all the searches. 
Table 2. Expected and observed number of isolated electron, muon, and tau events of H1 and ZEUS.

\begin{tabular}{|c|c|c|c|c|c|c|}
\hline & \multicolumn{2}{|c|}{ electron } & \multicolumn{2}{|c|}{ muon } & \multicolumn{2}{|r|}{ new } \\
\hline & all & $\mathrm{PT}^{\mathrm{x}}>25 \mathrm{GeV}$ & all & $\mathrm{PT}^{\mathrm{x}}>25 \mathrm{GeV}$ & all & $\mathrm{Pr}^{\mathrm{x}}>25 \mathrm{GeV}$ \\
\hline $\begin{array}{l}\text { H1 HERA I } \\
118 \mathrm{pb}^{-1}\end{array}$ & $\begin{array}{l}11 \\
11.54 \pm 1.50\end{array}$ & $\begin{array}{l}5 \\
1.76 \pm 0.30\end{array}$ & $\begin{array}{l}8 \\
2.94 \pm 0.50\end{array}$ & $\begin{array}{l}6 \\
1.68 \pm 0.30\end{array}$ & $\begin{array}{l}5 \\
5.8 \pm 1.36\end{array}$ & $\begin{array}{l}0 \\
0.53 \pm 0.10\end{array}$ \\
\hline $\begin{array}{l}H 1 e^{+} p \\
53 \mathrm{pb}^{-1}\end{array}$ & 9 & $\begin{array}{l}5 \\
0.84 \pm 0.19\end{array}$ & $\begin{array}{l}1 \\
1.33 \pm 0.19\end{array}$ & $\begin{array}{l}0 \\
0.85 \pm 0.13\end{array}$ & & \\
\hline $\begin{array}{l}H 1 e^{-p} \\
39 \mathrm{pb}^{-1}\end{array}$ new & $\begin{array}{l}5 \\
4.09 \pm 0.61\end{array}$ & $\begin{array}{l}1 \\
0.62 \pm 0.11\end{array}$ & $\begin{array}{l}0 \\
1.10 \pm 0.17\end{array}$ & $\begin{array}{l}0 \\
0.67 \pm 0.11\end{array}$ & & \\
\hline $\begin{array}{l}\text { ZEUS HERA I } \\
130 \mathrm{pb}^{-1}\end{array}$ & $\begin{array}{l}24 \\
20.6^{+1.7}{ }_{-4.6}\end{array}$ & $\begin{array}{l}2 \\
2.90^{+0.59}-0.32\end{array}$ & $\begin{array}{l}12 \\
11.9+0.6-0.7\end{array}$ & $\begin{array}{l}5 \\
2.75 \pm 0.21\end{array}$ & $\begin{array}{l}3 \\
0.40^{+0.12}-0.13\end{array}$ & $\begin{array}{l}2 \\
0.20 \pm 0.05\end{array}$ \\
\hline $\begin{array}{l}\text { ZEUS e+p new } \\
40 \mathrm{pb}^{-1}\end{array}$ & $\begin{array}{l}0 \\
0.46 \pm 0.10\end{array}$ & $\begin{array}{l}0 \\
0.58^{+0.08}-0.09\end{array}$ & & & & \\
\hline
\end{tabular}

\section{High Mass Searches}

High-mass searches were one of the first results presented from Run II of the Tevatron. New gauge bosons and other high mass resonances yield energetic objects when they decay. Searches based on energetic leptons, photons, and missing $E_{\mathrm{T}}$ give access to a large variety of new physics. For instance, events with an energetic electron and positron are sensitive to Z', large extra dimensions, Randall-Sundrum gravitons, $\not_{\mathrm{P}}$ sneutrinos, and technicolor particles, $\rho$ and $\omega$. The analyses ${ }^{25}$ of CDF and D $\varnothing$ are constantly refined, on one side to cover signatures in a generic way, by for instance calculating sensitivity based on the spin, or to incorporate new models and interpretations, like expressing Z' sensitivity based on $\mathrm{d}-x \mathrm{u}$, or $\mathrm{B}-x \mathrm{~L}$ couplings, on the other side to include additional event kinematics like $\cos \theta^{*}$ in the analysis to enhance sensitivity to new physics. About $450 \mathrm{pb}^{-1}$ of Run II data are analysed for high mass objects. No excess or deviation are observed so far.

\section{$7 \quad$ Indirect Searches}

With no signals of new physics in any of the direct searches, we can search for signs of new physics where new particles are in virtual states. Processes that are rare in the SM provide an excellent place to search for

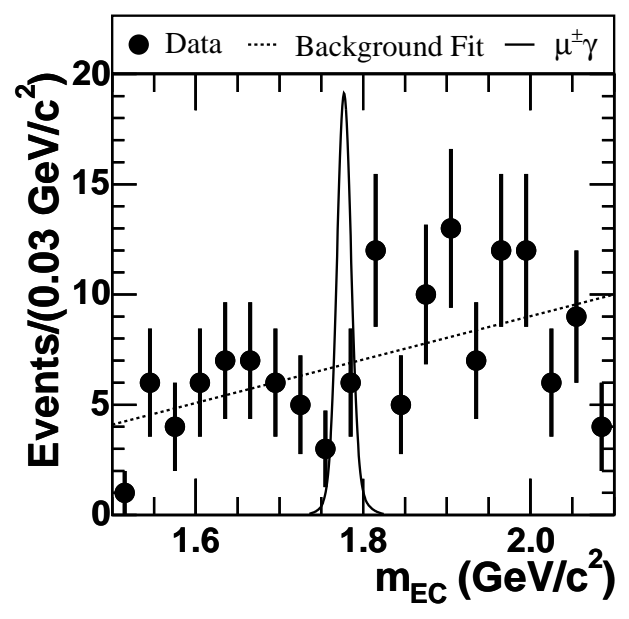

Figure 15. The energy constraint muon photon mass spectrum of the BaBar tau decay into muon plus photon analysis.

signs of new physics.

Tau decays into a muon and a photon are tiny in the SM with a branching ratio around $10^{-40}$ but allowed if one includes neutrino mixing. The decay violates lepton flavour which occures naturally in SUSY grand unified theories. Both Belle and $\mathrm{BaBar}^{26}$ have recorded over 20 million ditau events. BaBar uses one tau as tag and then the other as probe. A neural network is used to discriminate signal from background. The main background comes from dimuon production and ditau production with tau decays into a muon plus neutrinos and a photon from initial or 


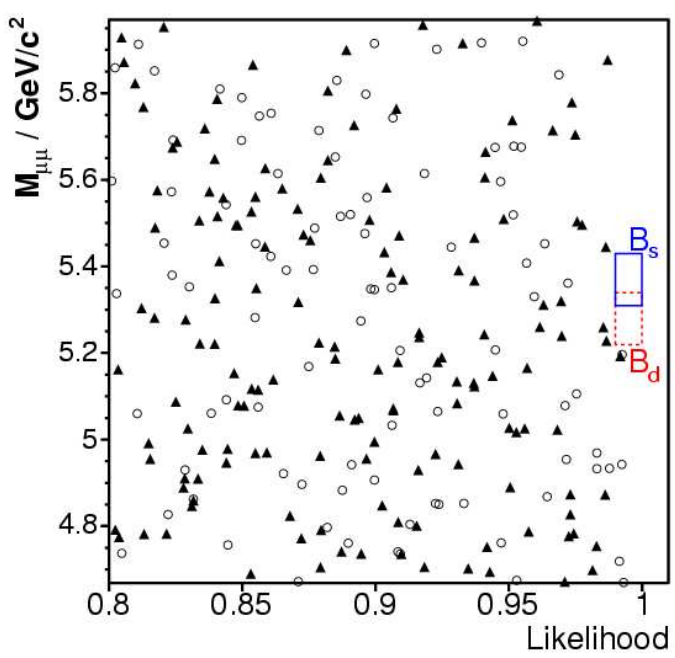

Figure 16. Distribution of events in the likelihood versus dimuon mass plane of the CDF $\mathrm{B} \rightarrow \mu^{+} \mu^{-}$ analysis.

final state radiation. Figure 15 shows the energy constraint muon photon mass with a curve of how a potential signal would look. Observation agrees with the background expectation and BaBar sets a 90\% CL limit on the branching ratio of tau into a muon plus a photon at $6.8 * 10^{-8}$.

Another interesting channel is the $B_{S}$ into $\mu^{+} \mu^{-}$decay. The flavor changing neutral current (FCNC) decay is heavily suppressed in the SM. In the MSSM, however, the branching ratio is enhanced, proportional to $\tan (\beta)^{6}$. CDF has a long tradition of searching for $\mathrm{B} \rightarrow \mu^{+} \mu^{-}$. The analysis is normalized to the observed $\mathrm{B}^{+} \rightarrow \mathrm{J} / \psi \mathrm{K}^{+}$decays to become independent of the $\mathrm{b}$ production cross-section. A likelihood function is used to separate dimuons that originate from a decay of a particle with lifetime from prompt dimuons. CDF observes no events in the $\mathrm{B}_{\mathrm{d}}$ and $\mathrm{B}_{\mathrm{s}}$ window, Fig. 16. The combined CDF/DØ analyses set a 95\% CL branching ratio limit of $1.2 * 10^{-7}$ for $\mathrm{B}_{\mathrm{S}}$ and $3.1 * 10^{-8}$ for $\mathrm{B}_{\mathrm{d}}$. This excludes first regions in SUSY parameter space at high $\tan (\beta)$.

\section{Summary and Outlook}

Scientists have explored nature to smaller and smaller scales over the years. In the last 50 years particle physics has made tremendous progress, revealing and exploring the next smaller layer of particles. We have developed a self-consistent, although incomplete, model that describes our current knowledge. Nature still surprises us, like with the observation of neutrino oscillation and the accelerating expansion of the universe. Our current understanding strongly suggests new physics to be close to the electroweak scale. However, no significant evidence of new physics has been observed so far. The current experiments search extensively in a large variety of signatures for deviations from the Standard Model. Some of the most interesting and promising search channels were presented in this review. Both HERA and the Tevatron are running well with record luminosities and the experiments are keeping up analysing the data. The hope is on the current experiments to unveil the next layer or the next symmetry of nature. A new generation of experiments is only a few years away and should answer our question about new electroweak scale physics. The transfer of expertise and experience to those new experiments has started.

\section{References}

1. P.W. Higgs, Phys. Lett. 12, 132 (1964); F. Englert and R. Brout, Phys. Rev. Lett. 13, 321 (1964);

G.S. Guralnik, C.R. Hagen, and T.W. Kibble, Phys. Rev. Lett. 13585 (1964).

2. K.A. Olive, (ed.), S. Rudaz, (ed.), M.A. Shifman, (ed.) Nucl. Phys. Proc. Suppl. 1011 (2001).

3. A. Juste, these proceedings.

4. S. Heinemeyer and G. Weiglein, Proceedings of the LCWS2000, hep-ph/0012364 (2000).

5. G. Abbiendi et al. Phys. Lett. B 565, 61 (2003).

6. For the most updated results please use the Higgs and New Particle Searches web site of 
the experiments at:

http://www-cdf .fnal.gov/physics/exotic /exotic.html and http://www-d0.fnal.go v/Run2Physics/higgs/pubresults.html

7. DØ Collab., DØnote 4774-CONF (2005).

8. Physics at Run II, Fermilab-PUB-00/349 (2000); CDF Collab., CDF note 6353 (2003)

9. ATLAS Collab., CERN/LHCC 99-15, ATLAS-TDR-15 (1999);

CMS Collab., CERN/LHCC 97-33, CMSTDR-4 (1997);

F. Gianotti, these proceedings.

10. A. Abulencia et al. hep-ex/0508051 (2005).

11. V.M. Abazov et al. hep-ex/0504018 (2005).

12. LEP Higgs Working Group, LHWG-Note 2005-01 (2005), paper 249 submitted to this conference.

13. M. Carena, J.R. Ellis, A. Pilaftsis, and C.E. Wagner Phys. Lett. B 495155 (2000); S. Heinemeyer, W. Hollik, and G. Weiglein Comp. Phys. Comm. 12476 (2000);

M. Frank, S. Heinemeyer, W. Hollik, and G. Weiglein, hep-ph/0212037 (2002).

14. CDF Collab., CDF note 7712 (2005).

15. LEP SUSY Working Group, LEPSUSYWG/0107.1 (2002).

16. V.M. Abazov et al. hep-ex/0504032 (2005); DØ Collab., DØnote 4740-CONF (2005).

17. CDF Collab., CDF note 7136 (2004); CDF Collab., CDF note 7457 (2005); DØ Collab., DØnote 4832-CONF (2005).

18. DØ Collab., DØnote 4737-CONF (2005).

19. A. Aktas et al. Eur. Phys. J. C 36425 (2004);

ZEUS Collab., ZEUS-prel-05-002, paper 258 submitted to this conference.

20. D. Acosta et al. Phys. Rev. D 71 031104(R) (2005);

V.M. Abazov et al. Phys. Rev. Lett. 94, 041801 (2005);

V. Buscher et al. hep-ex/0504004 (2005).

21. ATLAS Collab., CERN/LHCC 99-15 (1999);

M. Dittmar, CMS CR-1999/007 (1999);

A. Tricomi, CMS CR-2004/019 (2004);

22. J.L. Feng and M.M. Nojiri, hep-ph/0210390 (2002).

23. C. Adloff et al. Eur. Phys. J. C 5575 (1998);

V. Andreev et al. Phys. Lett. B 561241 (2003).

24. H1 Collab., paper 421 submitted to this con- ference;

H1 Collab., H1prelim-04-061, paper 417 submitted to this conference;

J. Breitweg et al. Phys. Lett. B 471, 411 (2000);

S. Chekanov et al. Phys. Lett. B 58341 (2004);

ZEUS Collab., paper 257 submitted to this conference.

25. CDF Collab., CDF note 7711 (2005); CDF Collab., CDF note 7286 (2004); CDF Collab., CDF note 7167 (2004); CDF Collab., CDF note 7098 (2004); CDF Collab., CDF note 7459 (2005); DØ Collab., DØnote 4336-CONF (2004); DØ Collab., DØnote 4922-CONF (2005); DØ Collab., DØnote 4552-CONF (2004); DØ Collab., DØnote 4893-CONF (2005); DØ Collab., DØnote 4829-CONF (2005).

26. BaBar Collab., hep-ex/0502032, paper 217 submitted to this conference; BaBar Collab., hep-ex/0409036 (2004); Belle Collab., hep-ex/0508044 (2005).

27. CDF Collab., hep-ex/0508036 (2005); DØ Collab., D0-Note 4733-Conf (2005). 


\section{DISCUSSION}

Inti Lehmann (Uppsala University):

What is the long term plans for the Tevatron after LHC comes into operation?

Stephan Lammel: As long as the Tevatron is at the energy frontier it would not be wise to switch off the machine. Once LHC has taken over one has to see if any measurments remain that can be done better at the Tevatron or if it is better to focus on LHC and may be prepare at Fermilab for a new experiment/machine.

Dieter Zeppenfeld (Uni. Karlsruhe):

With about $300 p b^{-1}$ or $5-10 \%$ of the ultimate data sample the Tevatron Higgs cross section bounds are more than an order of magnitude above SM expectations. This indicates that the present sensitivity is well below what was predicted. Is this correct? And what are the main problems?

Stephan Lammel: At the moment there is more than an order of magnitude difference between experimental limits and theoretical expectation. The important ingredients for Higgs search at the Tevatron are lepton and b-tagging efficiency. Both experiments are still improving on their detection efficiencies. The $\mathrm{b} \overline{\mathrm{b}}$ dijet mass resolution is also extremly important. Right now the experiments are back to their Run I mass resolution of $15 \%$ (from $17 \%$ at the start of Run II). The goal is to use b-jet specific corrections and global event variables which should bring the resolution to about $10 \%$. 